\title{
Genome-wide association study reveals a locus for nasal carriage of Staphylococcus aureus in Danish crossbred pigs
}

\author{
Per Skallerup ${ }^{1}$, Carmen Espinosa-Gongora², Claus B. Jørgensen ${ }^{1}$, Luca Guardabassi ${ }^{2}$ and Merete Fredholm*
}

\begin{abstract}
Background: Staphylococcus aureus is an important human opportunistic pathogen residing on skin and mucosae of healthy people. Pigs have been identified as a source of human colonization and infection with methicillinresistant Staphylococcus aureus (MRSA) and novel measures are needed to control zoonotic transmission. A recent longitudinal study indicated that a minority of pigs characterized by high nasal load and stable carriage may be responsible for the maintenance of $S$. aureus within farms. The primary objective of the present study was to detect genetic loci associated with nasal carriage of S. aureus in Danish crossbred pigs (Danish Landrace/Yorkshire/Duroc).

Results: Fifty-six persistent carriers and 65 non-carriers selected from 15 farms surveyed in the previous longitudinal study were genotyped using Illumina's Porcine SNP60 beadchip. In addition, spa typing was performed on 126 S. aureus isolates from 37 pigs to investigate possible relationships between host and S. aureus genotypes. A single SNP (MARC0099960) on chromosome 12 was found to be associated with nasal carriage of S. aureus at a genome-wide level after permutation testing $(p=0.0497)$ whereas the association of a neighboring SNP was found to be borderline ( $p=0.114)$. Typing of $S$. aureus isolates led to detection of 11 spa types belonging to the three main $S$. aureus clonal complexes (CC) previously described in pigs (CC9, CC30 and CC398). Individual carriers often harbored multiple S. aureus genotypes and the host-pathogen interaction seems to be independent of $S$. aureus genotype.
\end{abstract}

Conclusion: Our results suggest it may be possible to select pigs genetically resistant to $S$. aureus nasal colonization as a tool to control transmission of livestock-associated MRSA to humans.

Keywords: Pigs, Staphylococcus aureus colonization, MRSA control

\section{Background}

Staphylococcus aureus is a significant human pathogen causing wound and skin infections, endocarditis and bacteremia [1, 2]. It has long been recognized that healthy individuals may be colonized on skin and mucosae, the most frequent carriage site in humans being the anterior nares $[3,4]$. Longitudinal studies have demonstrated the existence of three S. aureus nasal carriage patterns, i.e., persistent carriers $(\sim 20 \%$ of the population), non-carriers $(\sim 50 \%)$ and intermittent carriers $(\sim 30 \%)[3,4]$. There is evidence showing that nasal carriage is associated with a higher risk of $S$. aureus infection $[5,6]$. Colonization of the host is a complex process

\footnotetext{
* Correspondence: mf@sund.ku.dk

${ }^{1}$ Department of Veterinary Clinical and Animal Sciences, University of

Copenhagen, Frederiksberg, Denmark

Full list of author information is available at the end of the article
}

which is influenced by host factors, bacterial factors, and environmental factors [6-8]. Although the heritability of $S$. aureus carriage was not significant in two human studies $[9,10]$, single nucleotide polymorphisms (SNPs) associated with nasal carriage have been found for several candidate genes, e.g., $\mathrm{C}$-reactive protein $(C R P)$, interleukin $4(I L-4)$, and glucocorticoid receptor (NR3C1) [11-15]. In addition, genetic loci associated with susceptibility to $\mathrm{S}$. aureus infection have been reported in murine studies [16, 17]. Collectively, these studies suggest that host gene variants underlie differences in susceptibility to $S$. aureus colonization. However, candidate gene studies suffer from the limitation that they only assess the effects of specific genes picked by the researcher based on hypothesized involvement in disease etiology. In contrast, genome-wide association studies (GWAS) survey the entire genome, 
and many of the associations found in GWAS identify novel candidate genes $[18,19]$.

Pigs and other livestock species carrying methicillinresistant S. aureus (MRSA) clonal complex (CC) 398 may act as a source of human colonization and infection $[20,21]$. Spread of this livestock-associated MRSA clone is presently regarded as a threat to public health, and effective control measures preventing transmission of MRSA to farmers and other people exposed to livestock are urgently needed [22, 23]. A recent longitudinal study revealed that a minority of pigs characterized by high nasal load and stable carriage may be responsible for the maintenance of S. aureus within farms [24]. The objective of the present study was to detect loci associated with nasal carriage of $S$. aureus. Danish crossbreds classified as persistent carriers $(n=56)$ and non-carriers $(n$ $=65$ ) were genotyped by GWAS using Illumina's Porcine SNP60 beadchip [25]. A single SNP on chromosome 12 was found to be genome-wide significant after permutation testing. The region of interest was inspected and we identified four candidate genes which may control $S$. aureus colonization in pigs.

\section{Methods}

\section{Phenotypic characterization of pigs}

Our study population comprised 56 persistent carriers and 65 non-carriers from 15 farms located in the central part of Jutland, Denmark (three specific-pathogen-free (SPF) and 12 non-SPF farms). Most pigs were phenotyped in the previous longitudinal study [24] and 21 additional pigs were recruited for this study on four of the farms surveyed in the longitudinal study. Nasal swabs $\left(\right.$ Dryswab $^{\text {tM }}$, MWE, UK) were collected from all pigs three times on a weekly basis. Pigs that were S. aureuspositive on all three sampling points were classified as persistent carriers whereas non-carriers were negative on at least two sampling points and with no more than $100 \mathrm{CFU} / \mathrm{swab}$ in the remaining sample. In order to ensure exposure to a minimum colonization pressure, noncarriers were included only if they originated from farms where at least one persistent carrier was detected. The distribution of persistent carriers and non-carriers among farms is shown in Additional file 1: Table S1. All pigs were crossbreeds (Danish Landrace/Yorkshire/ Duroc) of approximately $70 \mathrm{~kg}$. Pedigree details were not available but since the farmers used mixed semen to produce the offspring, the sample was expected to comprise a mixture of half-sibs (by sow and boar) and more distantly related pigs.

According to Danish laws (Danish Animal Experimentation Act, Chapter 1, Paragraph 1, point 3) no ethical approval was required for this study since the blood samples collected from the animals were taken for diagnostic purposes. All procedures concerning the animals were part of routine examinations and diagnosis of animals normally used at production farms. All handling of animals was performed by trained personnel and veterinarians.

\section{Genotyping of pigs}

To detect QTLs associated with $S$. aureus carrier status, we genotyped all pigs using diagnostic blood samples collected in EDTA tubes (VWR, USA). DNA was extracted using either a salting out procedure with minor

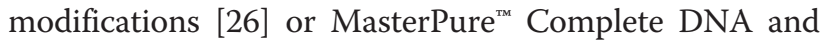
RNA Purification Kit (Epicentre Biotechnologies, USA) according to the manufacturer's instructions. The concentration and purity of DNA was measured on a NanoDrop 1000 spectrophotometer (Thermo Fisher Scientific, USA). $2500 \mathrm{ng}$ of each sample was submitted for genotyping to GeneSeek, Inc. (http://www.neogeneurope.com). Samples were genotyped for 61,565 SNPs using Illumina's Porcine SNP60 beadchip [25].

\section{Isolation and genotyping of $S$. aureus}

To study possible relationships between host and $S$. aureus genotypes, S. aureus was isolated from 37 persistent carriers whose nasal swabs had been stored at $-80{ }^{\circ} \mathrm{C}$ in the previous study [24]. Swabs were directly plated onto SaSelect agar (Biorad, USA) and enriched in Müller-Hinton broth containing $2.5 \%$ of $\mathrm{NaCl}$ to enhance $S$. aureus detection. After overnight incubation one presumptive $S$. aureus colony was randomly selected for each sampling point. Additional colonies were isolated if they had clear morphological features suggesting the presence of different strains on the same plate. If $S$. aureus was not detected by direct plating, the enrichments were further processed as described above. All isolates were characterized by spa typing [27, 28] and spa types were assigned using Ridom Staphtype software, version 2.2.1 (Ridom GmbH, Würzburg, Germany). Associations between spa types and clonal complexes (CC) were determined according to information available in the scientific literature. For spa types not previously associated to a clonal complex, BURP cluster analysis (Ridom StaphType software, version 2.2.1) was used to infer association [27].

\section{Statistical analyses}

Data were analyzed in $\mathrm{R}$ version 3.1.0 [29]. Genotype data were analyzed and visualized using the GenABEL package [30,31] except for Manhattan plots which were made using the qqman package [32]. SNPs were excluded prior to analysis if genome position was not provided $(n=12,627)$ or if they were located on sex chromosomes $(n=1381)$. SNP genotype data were subjected to quality control (QC) measures. GenABEL applies QC filters using an iterative process; for individuals we used the following criteria, call rates $>0.95$, false 
discovery rate (FDR) for unacceptably high heterozygosity $<0.01$ and identity-by-state (IBS) $<0.95$ (based on 2000 markers); for SNPs we used the following criteria (number of SNPs that did not pass the threshold), call rate $>0.95$ (2500), minor allele frequency (MAF) $>0.05$ (7012), and SNPs in Hardy-Weinberg equilibrium with $p$-values $>0.05(15,073)$. After QC a total of 23,919 autosomal SNPs mapped to build Sscrofa 10.2 and 121 individuals (56 carriers, 65 non-carriers) were included in the final analysis.

We estimated the average relatedness between pigs by computing an $n \times n$ marker-based genomic kinship matrix for all pairs of pigs. Kinship coefficients between two individuals (average identical-by-state value) were estimated using 23,919 autosomal SNPs which had passed QC as described elsewhere [31]. The genomic kinship matrix was transformed to a distance matrix which was then subjected to multidimensional scaling analysis and plotted in two dimensions (principal component axes) [31].

The association between SNP genotype and nasal carriage of $S$. aureus (binary trait) was tested in GenABEL using an allelic association test with $1 \mathrm{df}$. We included farm as a covariate in the model. To adjust for multiple testing with a high number of SNPs, we derived the empirical distribution of the chi-square statistic after 10,000 permutations of the whole dataset. Genome-wide significance was set to empirical $p$-values $<0.05$. We used genomic control [33] to adjust for any inflation of the test statistic. Calculations of linkage disequilibrium (LD) and visualization of LD were performed in Haploview version 4.2 [34]. Since the annotation of the pig genome sequence is incomplete, we also interrogated the human orthologue of the candidate region (HSA: 17q12) using builds Sscrofa10.2 and GRCh38 accessed through the Ensembl genome browser (www.ensembl.org).

The association between total number of spa types colonizing over the three-week period $(1,2$, or 3$)$ and SNP MARC0099960 genotype (GG, GA, AA) was tested using Fisher's Exact Test for Count Data. We tested if colonization by each clonal complex (CC9, CC30, and CC398) was non-random among SNP MARC0099960 genotypes using Fisher's Exact Test for Count Data. In addition to the genotype model, we also tested a dominance/recessive model (GG vs. GA and AA; GG and GA vs. AA).

\section{Results}

The 23,919 SNPs which passed quality testing were used to compute a genomic kinship matrix for all pairs of pigs (Fig. 1a). Inspection of the matrix suggested that the pigs in our sample were only distantly related. We next applied multidimensional scaling to a distance matrix calculated from the genomic kinship matrix (Fig. 1b). The plot did not suggest any stratification of data with respect to $S$. aureus carriage (persistent carriers vs. non-carriers) or farm (data not shown). We constructed a quantilequantile plot (Fig. 1c) and calculated the genomic inflation factor $\left(\lambda_{\mathrm{GC}}=1.06\right)$ which also confirmed that genetic confounding was not an issue in our dataset.

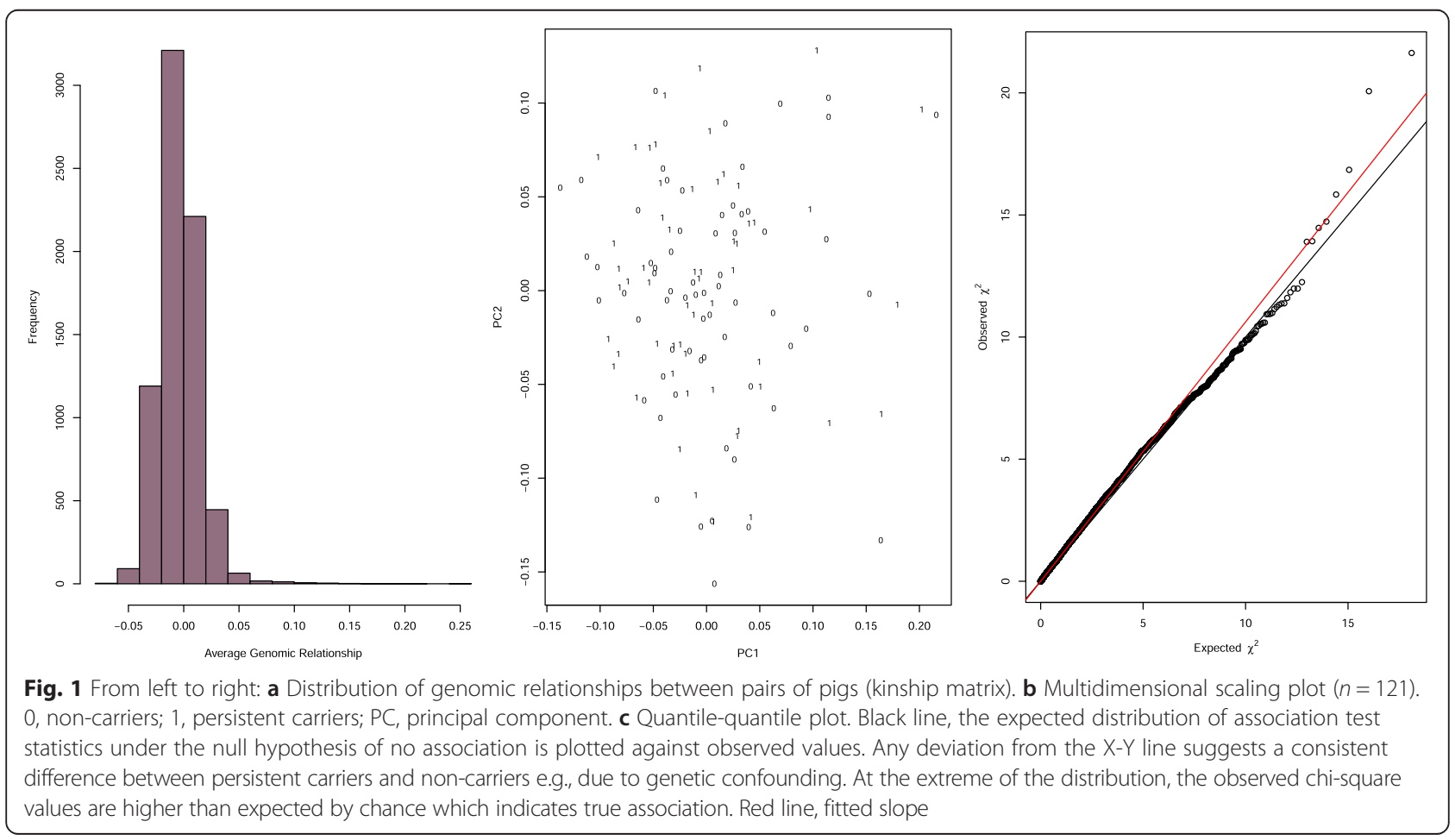


A GWAS was performed on the final dataset (Fig. 2). One locus (SNP MARC0099960) on porcine chromosome 12 demonstrated association with carriage of $S$. aureus. The effect was genome-wide significant after permutation testing $(p<0.05$; Table 1 ; Fig. 2$)$. A neighboring SNP, ALGA0104951, in high LD with MARC0099960 $\left(r^{2}=0.806\right)$, did not reach genomewide significance but was borderline significant after permutation testing (Table 1). These two SNPs are both located in an intergenic region.

To define the haplotype structure within the region, LD blocks were analyzed using Haploview. The measures of pairwise LD are shown in Fig. 3 where Block 1 indicates a region of $234 \mathrm{~kb}$ showing LD with SNP MARC0099960. The proposed candidate region is flanked by SNP markers ASGA0093685 and ALGA0123748, both showing no or weak LD with Block 1. Thus, the two SNPs (MARC0099960, ALGA0104951) delineate a haplotype block and since LD to flanking markers is weak we conservatively use these flanking markers as coordinates and delineate our QTL to SSC12: 42,422,021-43,436,573. With the limitations of the annotation of the porcine genome assembly, this QTL encompasses four annotated genes encoding chemokines (CCL1, CCL2, CCL8, CCL11).

The following 11 spa types were observed among the $126 \mathrm{~S}$. aureus isolates from persistent carriers (frequency in brackets): t011 (11\%), t034 (76 \%), t337 (5\%), t1333 (16\%), t1334 (8 \%), t1580 (3\%), t2315 (16\%), t2370
(3\%), t2462 (11\%), t3131 (14\%), and t5817 (5\%). Thirteen pigs $(35 \%)$ were found to carry the same strain throughout the study, while the remaining 24 pigs carried either two (59\%) or three (5\%) different strains (Additional file 1: Table S1). The identified spa types have previously been associated with CC9 (t337, t1334, t2315, t2462, t3131) [35-37], CC30 (t1333) [38] and CC398 (t011, t034, t1580, t2370) [35, 39]. We did not find any reported clonal complex association for spa type t5817, which belonged to CC9 according to BURP cluster analysis. The association between SNP MARC0099960 and host colonization was independent of $S$. aureus genotype.

\section{Discussion}

To our knowledge the work presented here is the first attempt to decipher the host genetic factors involved in nasal carriage of $S$. aureus in pigs. The GWAS demonstrated a significant association between a SNP marker located in a non-coding region (SNP MARC0099960) and nasal carriage of $S$. aureus, while the association of a nearby polymorphism in high LD with SNP MAR C0099960 (SNP ALGA0104951) was borderline significant. Regardless, the results presented here are preliminary and the association should be replicated in other pigs to confirm the findings.

The frequency of the $G$ allele at the SNP MAR C0099960 locus was twice as high in carrier pigs
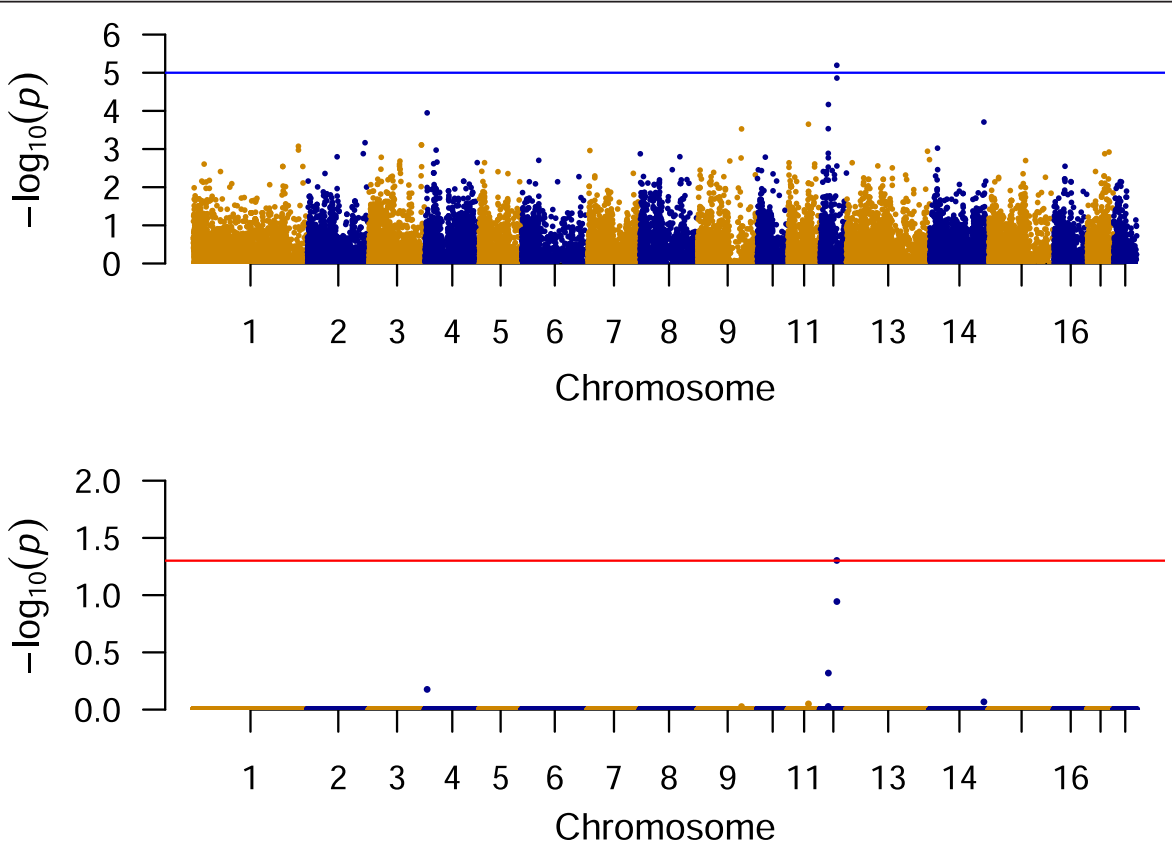

Fig. 2 Manhattan plots for GWAS of nasal Staphylococcus aureus carriage in Danish crossbred pigs. The analysis included 65 non-carriers and 56 persistent carriers which were genotyped at 23,919 autosomal SNPs. $p$-values were corrected using lambda statistic to account for genetic confounding. Upper figure shows raw $p$-values; a suggestive significance threshold $\left(p=1 \times 10^{-5}\right.$ is indicated with a horizontal line. Lower figure shows permuted dataset after 10,000 permutations; the horizontal line shows the genome-wide significance threshold $(p=0.05)$ 
Table 1 Statistics for two lead single nucleotide polymorphisms (SNPS) associated with nasal carriage of Staphylococcus aureus in Danish crossbred pigs (56 persistent carriers and 65 non-carriers)

\begin{tabular}{|c|c|c|c|c|c|c|c|}
\hline SNP ID & Chromosome & Position (bp) & $\mathrm{A} 1 / \mathrm{A} 2$ & MAF (persistent carriers) & MAF (non-carriers) & $p^{a}$ & $P^{b}$ \\
\hline MARC0099960 & 12 & $43,145,785$ & $\mathrm{G} / \mathrm{A}$ & 0.63 & 0.32 & $6.37 \times 10^{-6}$ & 0.0497 \\
\hline ALGA0104951 & 12 & $43,380,247$ & $\mathrm{G} / \mathrm{T}$ & 0.61 & 0.33 & $1.38 \times 10^{-5}$ & 0.1135 \\
\hline
\end{tabular}

MAF minor allele frequency; $A 1$ minor allele; $A 2$ major allele

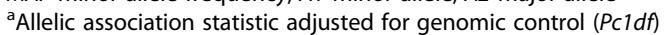

${ }^{b}$ Permutation test statistic after 10,000 permutations adjusted for genomic control

compared to non-carriers of S. aureus (Table 1). The majority (31 out of 36) of the 121 genotyped pigs that were homozygous for the A allele were non-carriers of $S$. aureus while the majority (20 out of 28 ) that were homozygous for the $\mathrm{G}$ allele were persistent carriers, suggesting that the $G$ allele is associated with susceptibility to nasal carriage of $S$. aureus. A total of 31 and 26 pigs were heterozygotes in the group of persistent- and non-carriers, respectively. Since both SNPs are located in a non-coding part of the genome, our results indicate that the haplotype tagged by these two SNPs contains one or several genes with an effect on S. aureus nasal carriage.

We used the Ensembl genome browser to interrogate our region of interest (build Sscrofa10.2) and the human orthologue of the region of interest (build GRCh38). Inspection of our $\sim 1 \mathrm{Mb}$ QTL region revealed that it encompasses a cluster of four chemokine genes (CCL1, $C C L 2, C C L 8$, and CCL11). A causative variant may be a SNP located in an exon of a protein-coding gene (changing the amino acid sequence of the protein), a regulatory part of a gene (altering the expression level), or a copy-number variant of a gene $[40,41]$. Chemokines are expressed by a variety of cells to help direct immune cells of the innate and adaptive branch of the immune system to the site of foreign antigen [42]. There is evidence suggesting some of our candidate chemokines may be invoked following bacterial colonization; S. aureus antigens have been shown to stimulate expression of CCL1 by dendritic cells [43] while another study demonstrated human alveolar epithelial cells produced CCL2 following stimulation by LPS, a component of the gramnegative cell wall [44]. CCL2 has chemotactic properties for monocytes [45]; indeed, recruitment of macrophages required expression of the CCL2-binding chemokine receptor 2 in a mouse model of Steptococcus pneumoniae colonization [46]. CCL1 and CCL11 had direct antimicrobial activity against $S$. aureus while CCL2 and CCL8 did not have any effect on this pathogen [47].

The host factors underlying the differences in S. aureus carriage are not yet fully understood [6]. Studies in a murine model have suggested $S$. aureus clearance is T-cell mediated and happens via an IL-17A-dependent recruitment of neutrophils [48]. While the adaptive immune response was found to be important, these authors were not able to demonstrate that B-cells were crucial. In agreement with these findings there is evidence showing that immunity to pneumococcal

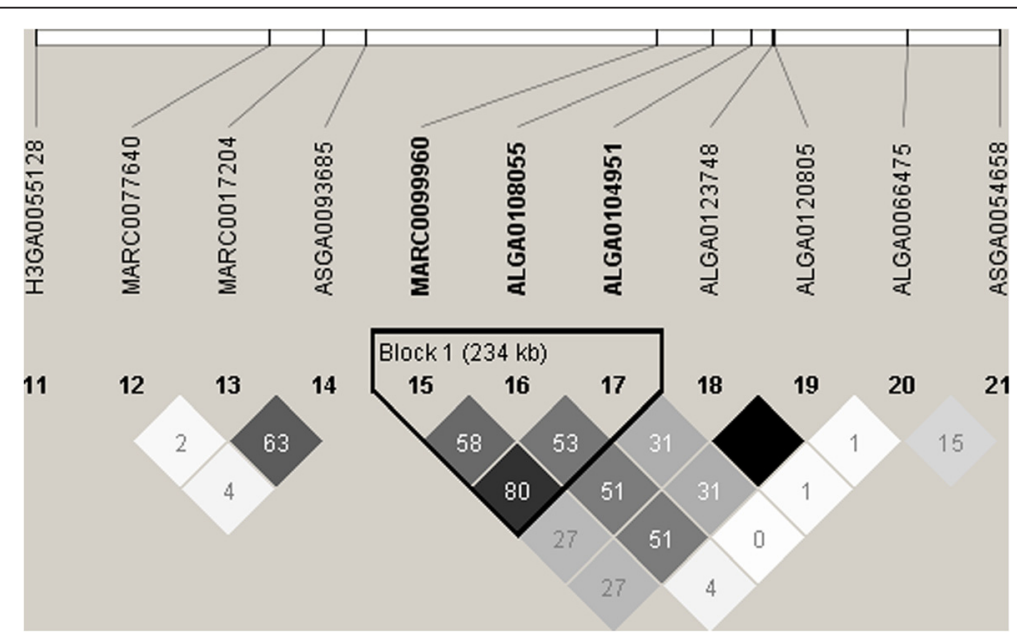

Fig. 3 Linkage disequilibrium (LD) plot showing $r^{2}$ x 100-values (correlation coefficient) with standard Haploview color scheme (ranging from white when $r^{2}=0$ to black when $r^{2}=1$ ). Block 1 indicates a region of 234 kb showing LD with SNP MARC0099960. The proposed candidate region is flanked by SNP markers ASGA0093685 and ALGA0123748, both showing no or week LD to block 1 
colonization is antibody independent but does require $\mathrm{CD}^{+} \mathrm{T}$ cells [49].

Research in humans and murine models using infection with $S$. aureus as phenotypic trait have suggested different positional candidate genes, e.g., SEH1L, TNFAIP8, KLK, and CDON [16, 17, 50, 51]. However, none of these genes are situated in or close to our QTL region. Genetic studies in human populations are challenged by a considerable genetic heterogeneity which may explain why previous efforts have shown a nonsignificant heritability of nasal carriage of S. aureus [9, 10]. In contrast, pigs may be used as a convenient model since they are much less heterogeneous and smaller sample sizes are needed to detect genetic variants associated with complex traits [52]. The pig model may be used to further explore $S$. aureus colonization mechanisms in humans; e.g., by taking advantage of the possibility to control various factors under experimental settings (e.g., housing conditions, known inoculation doses, known pedigrees, etc.).

GWAS for host susceptibility to infectious pathogens should take the genome of the microorganism, i.e., strain information, into account [53]. This notion is particularly relevant to $S$. aureus colonization, which is the result of a complex interplay between host and bacterial factors [54-56]. All S. aureus spa types identified in the present study have previously been isolated from pigs [35, 36, 38, 39] except t3131 (CC9) which has only been reported in cattle [37]. CC398-associated spa types were isolated from most (33/37) persistent carriers, suggesting that CC398 is the most prevalent $S$. aureus lineage in Danish crossbreed pigs. Even though the study was not designed to study coexistence of different lineages in the nasal cavity of pigs (i.e., only one or two isolates were spa typed from each sample), our results show that persistent carriers can be colonized by several lineages during a period of three weeks and by more than one strain at the same time.

The association between SNP MARC0099960 and host colonization was independent of $S$. aureus genotype. This is valuable information considering that $S$. aureus is a highly clonal microorganism and one clonal lineage (CC398) accounts for most livestock-associated MRSA infections in Europe [20, 21]. Identification of genetic markers associated with nasal carriage of $S$. aureus may be used in breeding to select animals with reduced susceptibility to colonization by this organism. Such a breeding program may serve as an unexplored option to prevent spread of livestock-associated MRSA to humans. A similar approach was used to detect the locus responsible for enterotoxigenic E. coli diarrhea in piglets, and a genetic marker test which distinguishes between susceptible and resistant animals has been developed [57]. The findings presented here may also improve our understanding of the host mechanisms underlying $S$. aureus colonization in both pigs and humans. The new locus detected in the present study provides a basis for further exploration by validation and functional testing of the markers and candidate genes.

\section{Conclusion}

We have identified significant association between a SNP marker located in a non-coding region (SNP MARC0099960) and nasal carriage of $S$. aureus. The QTL region encompasses a cluster of four chemokine genes (CCL1, CCL2, CCL8, and CCL11) which are potential candidate genes for nasal carrige. Our results suggest it may be possible to select pigs genetically resistant to $S$. aureus nasal colonization as a tool to control transmission of livestock-associated MRSA to humans.

\section{Additional file}

Additional file 1: Table S1. Pig and spa type distribution across farms (PDF $16 \mathrm{~kb}$ )

\section{Abbreviations}

S. aureus: Staphylococcus aureus; CC: Clonal complex; MRSA: Methicillin-resistant Staphylococcus aureus.

\section{Competing interests}

The authors declare that they have no competing interests.

\section{Authors' contributions}

PS carried out the genetic studies, statistical analysis and drafted the manuscript. CEG carried out the bacteriological study. CBJ participated in the design of the study and discussion of results. MF and LG conceived of the study, participated in its design, discussion of results and helped to draft the manuscript. All authors read and approved the final manuscript.

\section{Acknowledgements}

The authors wish to thank Tina Neergaard Mahler, Christel Ammitzböll Halberg and Ditte Lundt Gamborg for excellent technical assistance.

\section{Author details}

${ }^{1}$ Department of Veterinary Clinical and Animal Sciences, University of Copenhagen, Frederiksberg, Denmark. ²Department of Veterinary Disease Biology, University of Copenhagen, Frederiksberg, Denmark.

Received: 7 July 2015 Accepted: 18 November 2015

Published online: 26 November 2015

\section{References}

1. Archer GL. Staphylococcus aureus: a well-armed pathogen. Clin Infect Dis. 1998;26(5):1179-81.

2. Foster AP. Staphylococcal skin disease in livestock. Vet Dermatol. 2012;23(4): 342-51. e63.

3. Williams RE. Healthy carriage of Staphylococcus aureus: its prevalence and importance. Bacteriol Rev. 1963;27:56-71.

4. Wertheim HF, Melles DC, Vos MC, van Leeuwen W, van Belkum A, Verbrugh HA, et al. The role of nasal carriage in Staphylococcus aureus infections. Lancet Infect Dis. 2005;5(12):751-62.

5. von Eiff C, Becker K, Machka K, Stammer H, Peters G. Nasal carriage as a source of Staphylococcus aureus bacteremia. N Engl J Med. 2001;344(1):11-6.

6. Brown AF, Leech JM, Rogers TR, McLoughlin RM. Staphylococcus aureus colonization: modulation of host immune response and impact on human vaccine design. Front Immunol. 2014;4:507. 
7. Cole AM, Tahk S, Oren A, Yoshioka D, Kim YH, Park A, et al. Determinants of Staphylococcus aureus nasal carriage. Clin Diagn Lab Immunol. 2001;8(6): 1064-9

8. Weidenmaier C, Kokai-Kun JF, Kristian SA, Chanturiya T, Kalbacher H, Gross $\mathrm{M}$, et al. Role of teichoic acids in Staphylococcus aureus nasal colonization, a major risk factor in nosocomial infections. Nat Med. 2004;10(3):243-5.

9. Roghmann MC, Johnson JK, Stine OC, Lydecker AD, Ryan KA, Mitchell BD, et al. Persistent Staphylococcus aureus colonization is not a strongly heritable trait in Amish families. PLoS One. 2011;6(2):e17368.

10. Andersen PS, Pedersen JK, Fode P, Skov RL, Fowler Jr VG, Stegger M, et al. Influence of host genetics and environment on nasal carriage of Staphylococcus aureus in Danish middle-aged and elderly twins. J Infect Dis. 2012;206(8):1178-84

11. Uitterlinden AG, Koper JW, Nouwen JL, Melles DC, Van Rossum EF, Van den Akker EL, et al. Staphylococcus aureus nasal carriage is associated with glucocorticoid receptor gene polymorphisms. J Infect Dis. 2006;194(6):814-8.

12. van Belkum A, Emonts M, Wertheim H, de Jongh C, Nouwen J, Bartels $H$, et al. The role of human innate immune factors in nasal colonization by Staphylococcus aureus. Microbes Infect. 2007;9(12-13):1471-7.

13. Emonts M, Uitterlinden AG, Nouwen JL, Kardys I, Maat MP, Melles DC, et al. Host polymorphisms in interleukin 4, complement factor $\mathrm{H}$, and C-reactive protein associated with nasal carriage of Staphylococcus aureus and occurrence of boils. J Infect Dis. 2008;197(9):1244-53.

14. Vuononvirta J, Toivonen L, Grondahl-Yli-Hannuksela K, Barkoff AM, Lindholm L, Mertsola J, et al. Nasopharyngeal bacterial colonization and gene polymorphisms of mannose-binding lectin and toll-like receptors 2 and 4 in infants. PLoS One. 2011;6(10):e26198.

15. Nurjadi D, Herrmann E, Hinderberger I, Zanger P. Impaired beta-defensin expression in human skin links DEFB1 promoter polymorphisms with persistent Staphylococcus aureus nasal carriage. J Infect Dis. 2013;207(4):666-74.

16. Ahn SH, Deshmukh H, Johnson N, Cowell LG, Rude TH, Scott WK, et al. Two genes on $\mathrm{A} / \mathrm{J}$ chromosome 18 are associated with susceptibility to Staphylococcus aureus infection by combined microarray and QTL analyses. PLoS Pathog. 2010;6(9):e1001088.

17. Johnson NV, Ahn SH, Deshmukh H, Levin MK, Nelson CL, Scott WK, et al. Haplotype association mapping identifies a candidate gene region in mice infected with Staphylococcus aureus. G3 (Bethesda). 2012;2(6):693-700.

18. Altshuler D, Daly MJ, Lander ES. Genetic mapping in human disease. Science. 2008;322(5903):881-8.

19. Manolio TA, Brooks LD, Collins FS. A HapMap harvest of insights into the genetics of common disease. J Clin Invest. 2008;118(5):1590-605.

20. Huijsdens XW, van Dijke BJ, Spalburg E, van Santen-Verheuvel MG, Heck ME, Pluister GN, et al. Community-acquired MRSA and pig-farming. Ann Clin Microbiol Antimicrob. 2006;5:26.

21. Moodley A, Stegger M, Bagcigil AF, Baptiste KE, Loeffler A, Lloyd DH, et al. spa typing of methicillin-resistant Staphylococcus aureus isolated from domestic animals and veterinary staff in the UK and Ireland. J Antimicrob Chemother. 2006;58(6):1118-23.

22. Broens EM, Graat EA, van der Wolf PJ, van de Giessen AW, van Duijkeren E, Wagenaar JA, et al. MRSA CC398 in the pig production chain. Prev Vet Med. 2011;98(2-3):182-9.

23. Porphyre T, Giotis ES, Lloyd DH, Stärk KD. A metapopulation model to assess the capacity of spread of meticillin-resistant Staphylococcus aureus ST398 in humans. PLoS One. 2012;7(10):e47504.

24. Espinosa-Gongora C, Dahl J, Elvstrom A, van Wamel WJ, Guardabassi L. Individual predisposition to Staphylococcus aureus colonization in pigs based on quantification, carriage dynamics and serological profiles. Appl Environ Microbiol. 2015;81(4):1251-6.

25. Ramos AM, Crooijmans RP, Affara NA, Amaral AJ, Archibald AL, Beever JE, et al. Design of a high density SNP genotyping assay in the pig using SNPS identified and characterized by next generation sequencing technology. PLoS One. 2009;4(8):e6524.

26. Miller SA, Dykes DD, Polesky HF. A simple salting out procedure for extracting DNA from human nucleated cells. Nucleic Acids Res. 1988;16(3):1215.

27. Harmsen D, Claus H, Witte W, Rothganger J, Claus H, Turnwald D, et al. Typing of methicillin-resistant Staphylococcus aureus in a university hospital setting by using novel software for spa repeat determination and database management. J Clin Microbiol. 2003;41(12):5442-8.

28. Shopsin B, Gomez M, Montgomery SO, Smith DH, Waddington M, Dodge DE, et al. Evaluation of protein A gene polymorphic region DNA sequencing for typing of Staphylococcus aureus strains. J Clin Microbiol. 1999;37(11):3556-63.
29. R Core Team. R. A language and environment for statistical computing. Vienna, Austria: R Foundation for Statistical Computing; 2014.

30. Aulchenko YS, Ripke S, Isaacs A, van Duijn CM. GenABEL: an R library for genome-wide association analysis. Bioinformatics. 2007;23(10):1294-6.

31. GenABEL project developers. GenABEL: genome-wide SNP association analysis. R package version 1.8-0. 2013. http://CRAN.R-project.org/ package $=$ GenABEL

32. Turner SD. qqman: an R package for visualizing GWAS results using Q-Q and Manhattan plots. biorXiv 2014. DOI: 10 1101/005165.

33. Devlin B, Roeder K. Genomic control for association studies. Biometrics. 1999:55(4):997-1004.

34. Barrett JC, Fry B, Maller J, Daly MJ. Haploview: analysis and visualization of LD and haplotype maps. Bioinformatics. 2005;21(2):263-5.

35. Aarestrup FM, Skov RL, Stegger M, Guardabassi L, Moodley A, Hasman H. spa type distribution in Staphylococcus aureus originating from pigs, cattle and poultry. Vet Microbio. 2010;141(3-4):326-31.

36. Vandendriessche S, Vanderhaeghen W, Larsen J, de Mendonca R, Hallin M, Butaye $P$, et al. High genetic diversity of methicillin-susceptible Staphylococcus aureus (MSSA) from humans and animals on livestock farms and presence of SCCmec remnant DNA in MSSA CC398. J Antimicrob Chemother. 2014;69(2):355-62.

37. Bergonier D, Sobral D, Fessler AT, Jacquet E, Gilbert FB, Schwarz S, et al. Staphylococcus aureus from 152 cases of bovine, ovine and caprine mastitis investigated by Multiple-locus variable number of tandem repeat analysis (MLVA). Vet Res. 2014;45(1):97.

38. Aarestrup FM, Cavaco LM, Pedersen K, Agersø Y, Hasman H. Study of methicillin resistant Staphylococcus aureus (MRSA) in Danish pigs at slaughter and in imported retail meat reveals a novel MRSA type in slaughter pigs. Vet Microbiol. 2012;157(1-2):246-50.

39. Köck R, Schaumburg F, Mellmann A, Köksal M, Jurke A, Becker K, et al. Livestock-associated methicillin-resistant Staphylococcus aureus (MRSA) as causes of human infection and colonization in Germany. PLoS One. 2013; 8(2):e55040.

40. Hindorff LA, Sethupathy P, Junkins HA, Ramos EM, Mehta JP, Collins FS, et al. Potential etiologic and functional implications of genome-wide association loci for human diseases and traits. Proc Natl Acad Sci U S A. 2009;106(23):9362-7.

41. Hill AV. Evolution, revolution and heresy in the genetics of infectious disease susceptibility. Philos Trans R Soc Lond B Biol Sci. 2012;367(1590):840-9.

42. Roy I, Evans DB, Dwinell MB. Chemokines and chemokine receptors: update on utility and challenges for the clinician. Surgery. 2014;155(6):961-73.

43. Gombert M, Dieu-Nosjean MC, Winterberg F, Bünemann E, Kubitza RC, Da Cunha $L$, et al. CCL1-CCR8 interactions: an axis mediating the recruitment of $T$ cells and Langerhans-type dendritic cells to sites of atopic skin inflammation. J Immunol. 2005;174(8):5082-91.

44. Thorley AJ, Ford PA, Giembycz MA, Goldstraw P, Young A, Tetley TD Differential regulation of cytokine release and leukocyte migration by lipopolysaccharide-stimulated primary human lung alveolar type II epithelial cells and macrophages. J Immunol. 2007;178(1):463-73.

45. Deshmane SL, Kremlev S, Amini S, Sawaya BE. Monocyte chemoattractant protein-1 (MCP-1): an overview. J Interferon Cytokine Res. 2009;29(6):313-26.

46. Davis KM, Nakamura S, Weiser JN. Nod2 sensing of lysozyme-digested peptidoglycan promotes macrophage recruitment and clearance of $S$. pneumoniae colonization in mice. J Clin Invest. 2011;121(9):3666-76.

47. Yang D, Chen Q, Hoover DM, Staley P, Tucker KD, Lubkowski J, et al. Many chemokines including CCL20/MIP-3alpha display antimicrobial activity. J Leukoc Biol. 2003;74(3):448-55.

48. Archer NK, Harro JM, Shirtliff ME. Clearance of Staphylococcus aureus nasal carriage is T cell dependent and mediated through interleukin-17A expression and neutrophil influx. Infect Immun. 2013;81(6):2070-5.

49. Malley R, Trzcinski K, Srivastava A, Thompson CM, Anderson PW, Lipsitch M CD4+ T cells mediate antibody-independent acquired immunity to pneumococcal colonization. Proc Natl Acad Sci U S A. 2005;102(13):4848-53.

50. Nelson CL, Pelak K, Podgoreanu MV, Ahn SH, Scott WK, Allen AS, et al. A genome-wide association study of variants associated with acquisition of Staphylococcus aureus bacteremia in a healthcare setting. BMC Infect Dis. 2014;14:83.

51. Ye Z, Vasco DA, Carter TC, Brilliant MH, Schrodi SJ, Shukla SK. Genome wide association study of SNP-, gene-, and pathway-based approaches to identify genes influencing susceptibility to Staphylococcus aureus infections. Front Genet. 2014;5:125. 
52. de Koning DJ, Archibald A, Haley CS. Livestock genomics: bridging the gap between mice and men. Trends Biotechnol. 2007;25(11):483-9.

53. de Bakker PI, Telenti A. Infectious diseases not immune to genome-wide association. Nat Genet. 2010;42(9):731-2

54. Nouwen J, Boelens $\mathrm{H}$, van Belkum A, Verbrugh $\mathrm{H}$. Human factor in Staphylococcus aureus nasal carriage. Infect Immun. 2004;72(11):6685-8.

55. van Belkum A, Verkaik NJ, de Vogel CP, Boelens HA, Verveer J, Nouwen JL, et al. Reclassification of Staphylococcus aureus nasal carriage types. J Infect Dis. 2009;199(12):1820-6.

56. Johannessen M, Sollid JE. Hanssen AM. Host- and microbe determinants that may influence the success of $\mathrm{S}$. aureus colonization. Front Cell Infect Microbiol. 2012;2:56.

57. Jorgensen CB, Cirera S, Anderson SI, Archibald AL, Raudsepp T, Chowdhary $B$, et al. Linkage and comparative mapping of the locus controlling susceptibility towards E. coli F4ab/ac diarrhoea in pigs. Cytogenet Genome Res. 2003;102(1-4):157-62.

Submit your next manuscript to BioMed Central and we will help you at every step:

- We accept pre-submission inquiries

- Our selector tool helps you to find the most relevant journal

- We provide round the clock customer support

- Convenient online submission

- Thorough peer review

- Inclusion in PubMed and all major indexing services

- Maximum visibility for your research 